\title{
USO DE PANELES DE LÁMINAS ESTANDARIZADAS PARA LA EVALUACIÓN DE COMPETENCIAS EN EL DIAGNÓSTICO MICROSCÓPICO DE MALARIA EN LA AMAZONÍA PERUANA
}

\author{
Ángel Rosas-Aguirre ${ }^{1,2, a, c}$, Dionicia Gamboa 2,b,e, Hugo Rodriguez ${ }^{1, a}$, Fernando Llanos-Zavalaga ${ }^{2, a, d}$, \\ Kristhian Aguirre ${ }^{3, a}$, Alejandro Llanos-Cuentas ${ }^{1,2, a, f}$
}

\section{RESUMEN}

Objetivos. Evaluar la competencia de los microscopistas en el diagnóstico de la malaria mediante paneles de láminas estandarizados en la Amazonía peruana. Materiales y métodos. Estudio transversal, realizado entre los meses de julio y septiembre de 2007, en 122 establecimientos de salud de primer nivel de atención de la Amazonía peruana. En el marco del Proyecto PAMAFRO, se evaluó las competencias en el diagnóstico de malaria en 68 microscopistas sin experiencia (<un año experiencia) y 76 microscopistas con experiencia (>un año), utilizando paneles estandarizados de 20 láminas siguiendo las recomendaciones de la Organización Mundial de la Salud (OMS). Se definió concordancia como el porcentaje de aciertos que tuvo el microscopista en los diagnósticos (identificación de especie del parásito). Un microscopista con concordancia $\geq 90 \%$ ( $\geq 18$ láminas acertadas) fue definido como "experto", 80 a $<90 \%$ "referente", 70 a $<80 \%$ "competente" y $<70 \%$ "en entrenamiento". Resultados. Los microscopistas con experiencia (68,6\%) tuvieron mayor concordancia que aquellos sin experiencia $(48,2 \%)$. La competencia fue aceptable (niveles competente, referente o expertos) en $11,8 \%$ de los microscopistas sin experiencia y en $52,6 \%$ de aquellos con experiencia. La concordancia fue menor para láminas con P. falciparum con parasitemia baja, con P. malariae y con infecciones mixtas. Conclusiones. En esta primera evaluación, se evidenció que solo uno de cada tres microscopistas de la amazonía peruana es competente para el diagnóstico de malaria según estándares de la OMS. A partir de esta línea de base, se debe mejorar las competencias de los microscopistas en el marco de un sistema de aseguramiento de la calidad.

Palabras clave: Malaria/diagnóstico; Competencia profesional; Microscopía; Control de calidad; Perú (fuente: DeCS BIREME).

\section{USE OF STANDARDIZED BLOOD SMEAR SLIDE SETS FOR COMPETENCY ASSESSMENT IN THE MALARIA MICROSCOPIC DIAGNOSIS IN THE PERUVIAN AMAZON}

\begin{abstract}
Objectives. To assess the competency of microscopists for malaria diagnosis using standardized slide sets in the Peruvian Amazon. Material and methods. Cross-sectional study carried out in 122 first level health facilities of the Peruvian Amazon, between July and September 2007. Within the frame of the project "Control Malaria in the border areas of the Andean Region: A community approach" (PAMAFRO), we evaluated the malaria diagnosis performance in 68 microscopists without expertise (<1 year of expertise) and 76 microscopists with expertise ( 1 year) using standardized sets of 20 blood smear slides according to the World Health Organization (WHO) recommendations. A correct diagnosis (correct species identification) was defined as "agreement", a microscopist was qualified as an "expert" if they have an agreement $\geq 90 \%$ ( $\geq 18$ slides with correct diagnosis), as a "referent" with an agreement between $80 \%$ and $<90 \%$, "competent" if they are between 70 and $<80 \%$ and "in training" if they have $<70 \%$. Results. Microscopists with expertise $(68.6 \%)$ had more agreement than those without expertise $(48.2 \%)$. The competency assessment was acceptable (competent, referent, or experts levels) in $11.8 \%$ of the microscopists without expertise and in $52.6 \%$ from those with expertise. The agreement was lower using blood smear slides with $P$. falciparum with low parasitaemia, with $P$. malariae and with mixed infections. Conclusions. Is the first assessment, we found only one of three microscopists from the Peruvian Amazon is competent fro malaria diagnosis according to the WHO standards. From this baseline data, we have to continue working in order to improve the competency assessment of the microscopists within the frame of a quality assurance system
\end{abstract}

Key words: Malaria/diagnosis; Professional competence; Microscopy; Quality control; Peru (source: MeSH NLM).

Proyecto PAMAFRO, Organismo Andino de Salud-Convenio Hipólito Unanue, Lima, Perú.

Universidad Peruana Cayetano Heredia, Lima, Perú.

Solidaridad en Marcha, Lima, Perú.

a Médico; b Bióloga; ' Magister en Salud Pública; d Magister en Políticas, planificación y financiamiento en Salud; e Doctor (PhD) en Biología Molecular Parasitaria; ${ }^{\mathrm{f}}$ Doctor $(\mathrm{PhD})$ en Epidemiología y Enfermedades Tropicales.

Recibido: 08-10-10 Aprobado: 07-12-10 


\section{INTRODUCCIÓN}

La malaria es la enfermedad parasitaria de mayor prevalencia en el mundo (1). En el Perú, es considerada un grave problema de salud pública, debido a su incidencia y extensión geográfica (aproximadamente 75\% del territorio), así como su alto costo social y económico (2). LaAmazonía (principalmente el departamento de Loreto) es el área de mayor transmisión de esta enfermedad, habiéndose reportado en el último quinquenio alrededor del 60 a $70 \%$ de casos del país, entre 30000 y 40000 casos por año ${ }^{(3)}$.

El primer componente de la estrategia global de control de la malaria es el acceso al diagnóstico y tratamiento apropiado y oportuno. La implementación de políticas que garanticen acceso a un tratamiento apropiado se fundamenta necesariamente en la existencia de un sistema de atención que ofrezca, con oportunidad, acceso a un diagnóstico confiable ${ }^{(4,5)}$.

El diagnóstico microscópico es el mejor método para la confirmación de la malaria. El cuidadoso examen de una buena preparación y fijación de un extendido grueso (gota gruesa) y uno fino (frotis) de una muestra de sangre sobre una lámina sigue siendo, en la actualidad, la "prueba de oro" para el diagnóstico de malaria (6-9). El procedimiento consiste en colectar una muestra de sangre del pulpejo del dedo, preparar la gota gruesa y el frotis sobre una lámina portaobjetos, aplicarle un colorante a la lámina (frecuentemente Giemsa) y examinarla con ayuda de un microscopio buscando la presencia de parásitos de la malaria ${ }^{(9,10)}$. Cualquier falla en este proceso afectaría el resultado de esta prueba de diagnóstico.

La Organización Mundial de la Salud (OMS) ha definido la competencia como la habilidad de un microscopista para lograr precisión en el diagnóstico cuando realiza el examen y reporte de una lámina de sangre con malaria. La OMS propone que se debe definir la habilidad específica requerida para cada nivel de un sistema de aseguramiento de la calidad. La propuesta busca la mejora del desempeño rutinario de los microscopistas (1113), teniendo como pilares principales: el establecimiento de estándares de competencia en el microscopista, el entrenamiento apropiado que lleve a la competencia deseada y la evaluación y acreditación de dicha competencia.

La evaluación y acreditación de la competencia debe ser estandarizada usando pruebas de proficiencia mediante paneles de láminas estandarizados (composición por parásito y parasitemia definida). De esta forma, se ha establecido una gradación para la valoración final de la competencia con porcentajes para la precisión en la identificación y cuantificación del parásito en la lámina ${ }^{(12,14)}$

Siguiendo las recomendaciones de la OMS, en el marco del Proyecto "Control de la Malaria en las Zonas Fronterizas de la Región Andina: Un Enfoque Comunitario" (PAMAFRO) que tiene financiamiento del "Fondo Mundial para el VIH-SIDA, Tuberculosis y Malaria", se viene fortaleciendo el sistema de gestión de calidad del diagnóstico de la malaria en el Perú, a través de la capacitación a los microscopistas, la evaluación de competencias y el mejoramiento en la eficiencia del monitoreo del desempeño periódico (control de calidad) ${ }^{(12-13)}$.

Durante los dos primeros años de ejecución, el proyecto PAMAFRO en asociación con el Instituto de Medicina Tropical Alexander Von Humboldt de la Universidad Peruana Cayetano Heredia implementó un programa de capacitación para microscopistas. En este artículo presentamos los resultados de la evaluación de competencias del personal a cargo del diagnóstico microscópico en los establecimientos de salud en los tres departamentos del ámbito de intervención del proyecto: Loreto (provincias de Maynas, Alto Amazonas, Datem, Loreto y Ramón Castilla), Cajamarca (provincias Jaén y San Ignacio) y Amazonas (provincia Condorcanqui).

\section{MATERIALES Y MÉTODOS}

Estudio observacional, descriptivo, de corte transversal, realizado durante los meses de julio a septiembre de 2007, en el que se evaluó las competencias en el diagnóstico microscópico de la malaria en 144 personas a cargo de esta actividad en 122 establecimientos de salud de los departamentos de Loreto (provincias Maynas, Alto Amazonas-Datem, Loreto-Ramón Castilla), Cajamarca (provincias Jaén-San Ignacio) y Amazonas (provincia Condorcanqui) en el Perú, los cuales forman parte del ámbito de intervención del Proyecto PAMAFRO. La Figura 1 presenta la ubicación de los departamentos en el territorio peruano.

Los microscopistas evaluados participaron previamente en el programa de capacitación en microscopía que implementó el proyecto PAMAFRO en asociación con el Instituto de Medicina Tropical Alexander Von Humboldt (IMTAvH) de la Universidad Peruana Cayetano Heredia (UPCH). Considerando la modalidad de participación y la experiencia de trabajo en el diagnóstico microscópico al momento de la evaluación de competencias, los microscopistas fueron clasificados en: a) microscopistas sin experiencia y b) microscopistas con experiencia. 


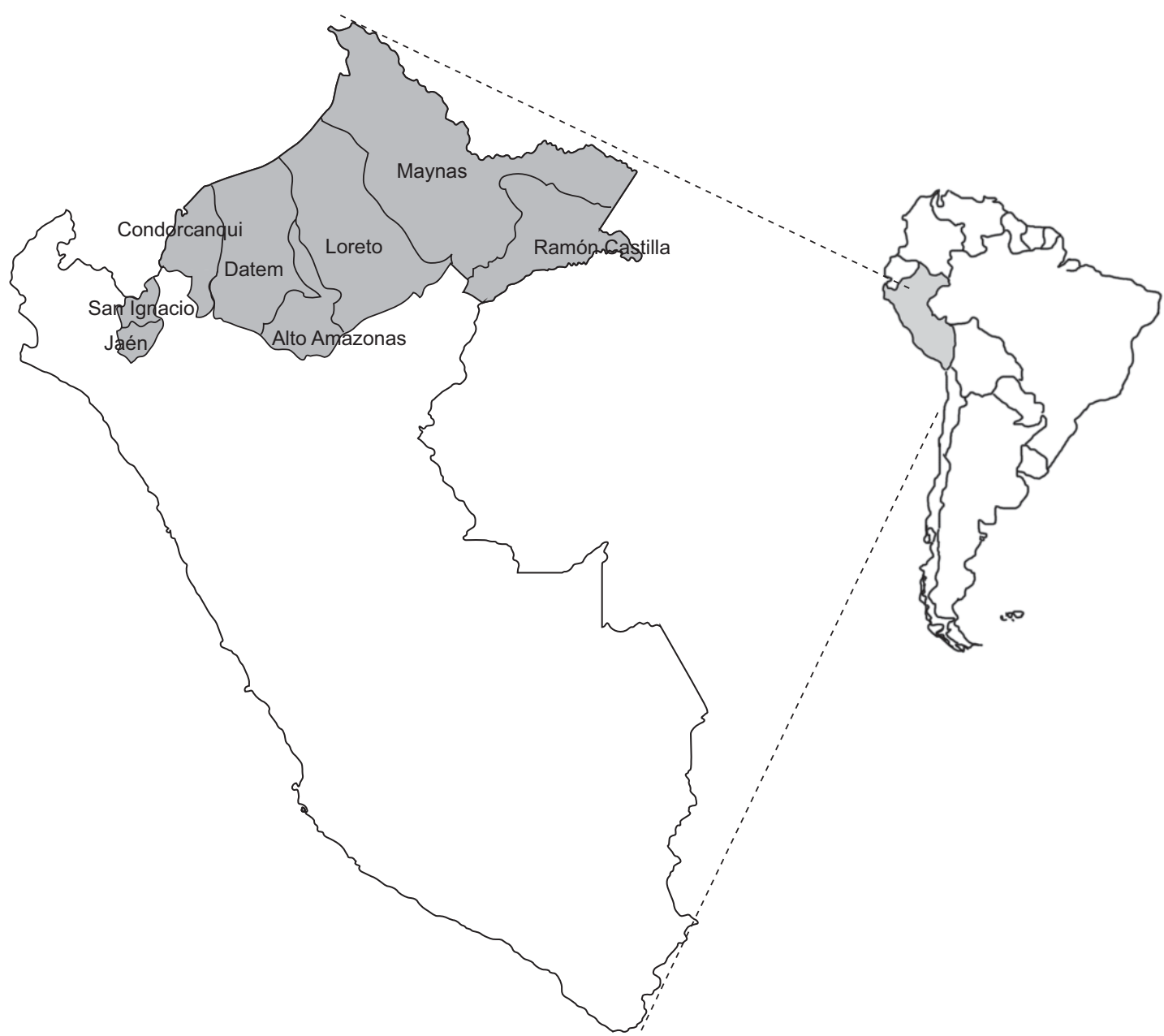

Figura 1. Ámbito geográfico para la evaluación de competencias en el diagnóstico microscópico de la malaria en la Amazonía peruana, 2007.

Los primeros eran, en su mayoría, técnicos de enfermería sin experiencia que fueron capacitados en microscopía durante en el primer semestre del 2007, y que al momento de la evaluación de competencias tenían menos de un año realizando diagnósticos microscópicos de malaria en sus establecimientos. La capacitación de ellos fue eminentemente práctica con dos fases presenciales (la primera de dos semanas y la segunda de una semana) y entre ambas, un periodo de cuatro semanas para la lectura de láminas en sus establecimientos de salud bajo la supervisión de facilitadores de la institución educativa a cargo del programa.

Los microscopistas con experiencia siempre estuvieron a cargo del diagnóstico microscópico en sus respectivos establecimientos y habían sido capacitados en el 2006 durante dos semanas en forma presencial, con un reforzamiento práctico de una semana también presencial en el primer semestre del 2007. Al momento de la evaluación de competencias, dichos microscopistas tenían más de un año de experiencia en el diagnóstico microscópico.

Para la evaluación de competencias, se utilizó paneles de láminas estandarizados, elaborados por el laboratorio de malaria del IMTAvH de la UPCH. En la preparación de los paneles de láminas se usó muestras de sangre obtenidas en tubos EDTA de pacientes con malaria y donantes sanos, quienes previamente habían firmado un consentimiento informado para el uso de las muestras con fines de investigación, con aprobación del Comité de Ética de la UPCH. La confirmación del diagnóstico de los donantes fue por microscopía y PCR. Todas las muestras fueron procesadas dentro de las seis horas de su colección.

Con ayuda de una micropipeta y puntas estériles, se colocaron dos gotas de sangre (una grande de $8 \mu \mathrm{L}$ 
en el tercio externo y una pequeña de $4 \mu \mathrm{L}$ en la parte central) sobre la superficie de una lámina biselada $(25 \mathrm{~mm} \times 75 \mathrm{~mm})$, permitiendo la preparación de la gota gruesa y el frotis, respectivamente, de acuerdo con las directrices nacionales para el diagnóstico de malaria ${ }^{(9)}$. En la coloración se usó un lote fresco de Giemsa al 10\% en solución buffer fosfato (PBS, pH 7,2).

Posteriormente, las láminas fueron almacenadas en cajas limpias de madera por una semana; luego de lo cual fueron montadas en bálsamo de Canadá y cubiertas por una lámina cubreobjetos para incrementar su vida útil. Las láminas fueron secadas al aire ambiente por 60 días, etiquetadas con códigos únicos de identificación encriptados y luego almacenadas en cajas plásticas (paneles).

Los paneles incluyeron 20 láminas preparadas con muestras de sangre con diferentes especies de Plasmodium y densidades parasitarias definidas. La composición de los paneles se ajustó a las recomendaciones de la OMS ${ }^{(11)}$, reemplazando las láminas de $P$. ovale y las láminas de presentación inusual por láminas con $P$. vivax, $P$. falciparum o malaria mixta. Los diagnósticos correctos (identificación de especie del parásito) de las láminas del panel solo eran conocidos por el responsable del laboratorio de malaria de la institución educativa ya mencionada. La Tabla 1 muestra la composición de los paneles.

Los microscopistas evaluados usaron sus propios microscopios (todos en buenas condiciones, la mayoría de ellos microscopios nuevos que fueron entregados por el proyecto unos meses antes) y la evaluación se llevó a cabo en sus propios establecimientos de salud siguiendo las pautas señaladas por la OMS: diez minutos por lámina, usar solo aceite de inmersión para lectura, usar materiales de ayuda y capacitación de ser necesario y mantener tranquilidad y silencio en el lugar de evaluación. Al final de la evaluación los microscopistas registraban los diagnósticos parasitológicos y la densidad parasitaria en una ficha de datos diseñada específicamente para la evaluación.

Se definió concordancia como el porcentaje de aciertos que tuvo el microscopista en los diagnósticos (identificación de especie del parásito) luego de la lectura de las 20 láminas. De acuerdo con las recomendaciones de la OMS ${ }^{(11)}$, se clasificó a los microscopistas según las siguientes categorías de competencia: a) "en entrenamiento" cuando la concordancia era menor de $70 \%$ (acierto en menos de 14 láminas en la identificación del parásito o diagnóstico), b) "competente" cuando la concordancia era mayor o igual a $70 \%$ pero menor de $80 \%$ (de 14 a 15 láminas acertadas), c) "referente" cuando la concordancia era mayor o igual a $80 \%$ pero menor de $90 \%$ (de 16 a 17 láminas acertadas), y d)
Tabla 1. Composición de los paneles de láminas usados durante la evaluación de competencias en el diagnóstico microscópico de la malaria en la Amazonía peruana, 2007.

\begin{tabular}{|c|c|c|c|c|}
\hline \multirow{2}{*}{$\begin{array}{l}\text { Diagnóstico } \\
\text { Densidad parasitaria }\end{array}$} & \multicolumn{4}{|c|}{ Cantidad de láminas } \\
\hline & Media & Mediana & Mínima & Máxima \\
\hline Negativos & 5,2 & 5 & 3 & 7 \\
\hline \multicolumn{5}{|l|}{$P$. vivax } \\
\hline$\geq 500 \mathrm{par} / \mu \mathrm{L}$ & 3,6 & 3 & 3 & 3 \\
\hline$<500$ par/ $/ \mu \mathrm{L}$ & 1,7 & 2 & 0 & 4 \\
\hline \multicolumn{5}{|l|}{ P. falciparum } \\
\hline$\geq 500 \mathrm{par} / \mu \mathrm{L}$ & 4,3 & 4,5 & 5 & 4 \\
\hline$<500 \mathrm{par} / \mu \mathrm{L}$ & 1,4 & 1,5 & 0 & 3 \\
\hline \multicolumn{5}{|l|}{ P. malariae } \\
\hline$\geq 500 \mathrm{par} / \mu \mathrm{L}$ & 1,5 & 2 & 0 & 2 \\
\hline \multicolumn{5}{|c|}{ Malaria mixta ( $P$. falciparum y $P$. vivax) } \\
\hline$\geq 500 \mathrm{par} / \mu \mathrm{L}$ & 0,9 & 1 & 2 & 3 \\
\hline$<500 \mathrm{par} / \mu \mathrm{L}$ & 1,3 & 1 & 0 & 2 \\
\hline Total & & 20 & & \\
\hline
\end{tabular}

"experto" cuando la concordancia era mayor o igual a $90 \%$ (de 18 a 20 láminas acertadas).

Los diagnósticos verdaderos, y los diagnósticos registrados por los microscopistas evaluados fueron ingresados a una hoja de cálculo en Excel. El análisis fue realizado usando el paquete estadístico $R$, calculándose las medias y medianas de las concordancias (expresadas en porcentajes) de los diagnósticos, en forma global o específica según provincia, experiencia del microscopista y lámina.

\section{RESULTADOS}

De los 144 microscopistas evaluados, 76 tenían más de un año de experiencia en microscopía. La mayoría (82) fueron del departamento de Loreto: 34 de la provincia de Maynas, 36 de las provincias Alto Amazonas y Datem, y 12 de las provincias Loreto y Ramón Castilla. El resto de microscopistas evaluados fueron de Cajamarca y Amazonas, 33 de las provincias Jaén y San Ignacio y 29 de la provincia de Condorcanqui, respectivamente.

La concordancia (porcentaje de aciertos en la identificación del parásito en las 20 láminas) fue mayor en los microscopistas con experiencia (media: $68,6 \%$, mediana: $70,0 \%$ ) cuando se comparó con los microscopistas sin experiencia (media: $48,2 \%$, mediana: $45,0 \%$ ). Los microscopistas con experiencia que tuvieron la mejor concordancia procedieron de las provincias Alto Amazonas-Datem, (media: 73,2\%, mediana: $75 \%$ ); 
Tabla 2. Concordancia en el diagnóstico (identificación del parásito) en microscopistas de la Amazonía Peruana, 2007.

\begin{tabular}{|c|c|c|c|c|c|c|c|c|c|}
\hline \multirow{3}{*}{$\begin{array}{l}\text { Departamento } \\
\text { Provincia }\end{array}$} & \multicolumn{3}{|c|}{$\begin{array}{l}\text { Microscopista sin } \\
\text { experiencia }\end{array}$} & \multicolumn{3}{|c|}{$\begin{array}{c}\text { Microscopista con } \\
\text { experiencia }\end{array}$} & \multicolumn{3}{|c|}{ Total microscopistas } \\
\hline & \multirow{2}{*}{$\mathbf{N}$} & \multicolumn{2}{|c|}{ Concordancia (\%) } & \multirow{2}{*}{$\mathbf{N}$} & \multicolumn{2}{|c|}{ Concordancia (\%) } & \multirow{2}{*}{$\mathbf{N}$} & \multicolumn{2}{|c|}{ Concordancia (\%) } \\
\hline & & Media & Mediana & & Media & Mediana & & Media & Mediana \\
\hline \multicolumn{10}{|l|}{ Loreto } \\
\hline Maynas & 21 & 43,6 & 40,0 & 13 & 68,8 & 65,0 & 34 & 53,2 & 52,5 \\
\hline Alto Amazonas - Datem & 17 & 59,7 & 65,0 & 19 & 73,2 & 75,0 & 36 & 66,8 & 67,5 \\
\hline Loreto-Ramón Castilla & 7 & 40,0 & 45,0 & 5 & 73,0 & 70,0 & 12 & 53,8 & 50,0 \\
\hline \multicolumn{10}{|l|}{ Cajamarca } \\
\hline Jaén - San Ignacio & 8 & 51,9 & 50,0 & 25 & 70,6 & 70,0 & 33 & 66,1 & 65,0 \\
\hline \multicolumn{10}{|l|}{ Amazonas } \\
\hline Condorcanqui & 15 & 43,7 & 50,0 & 14 & 57,1 & 55,0 & 29 & 50,2 & 50,0 \\
\hline Total & 68 & 48,2 & 45,0 & 76 & 68,6 & 70,0 & 144 & 59,0 & 60,0 \\
\hline
\end{tabular}

también la mejor concordancia en los microscopistas sin experiencia fue en ambas provincias (media: $59,7 \%$, mediana: 65,0\%) (Tabla 2). Los microscopistas con experiencia que tuvieron la menor concordancia procedieron de Condorcanqui (media: 57,1\%, mediana: $55,0 \%$ ) y los microscopistas sin experiencia con la menor concordancia procedieron también de la misma provincia (media: 43,7\%, mediana: 50,0\%), de Maynas (media: 43,6\%, mediana: 40,0\%) y de Loreto-Ramón Castilla (media: 40,0\%, mediana: 45,0\%).

La Tabla 3 presenta la clasificación de los microscopistas según la valoración de la competencia. La mayoría de microscopistas sin experiencia $(88,2 \%)$ fueron calificados como microscopistas "en entrenamiento", solo cinco $(7,4 \%)$ alcanzaron en nivel "competente", dos
$(2,9 \%)$ el nivel "referente" y uno (1,5\%) alcanzó concordancia de un "experto". Con relación a los microscopistas con experiencia, $36(47,4 \%)$ no alcanzaron concordancias aceptables siendo clasificados en el grupo "en entrenamiento", $14(18,4 \%)$ fueron considerados "competentes", $17(22,4 \%)$ "referentes" y $9(11,8 \%)$ "expertos". Como se presenta en la Figura 2, todos los microscopistas sin experiencia de Loreto-Ramón Castilla y de Condorcanqui fueron clasificados como microscopistas "en entrenamiento". Las provincias que tuvieron mayor porcentaje de microscopistas con experiencia "competentes", "referentes" o "expertos" fueron Alto Amazonas -Datem $(68,4 \%)$ y Jaén-San Ignacio $(60,0 \%)$.

Posteriormente, se realizó un análisis de la concordancia por cada lámina del panel con la intención de identificar

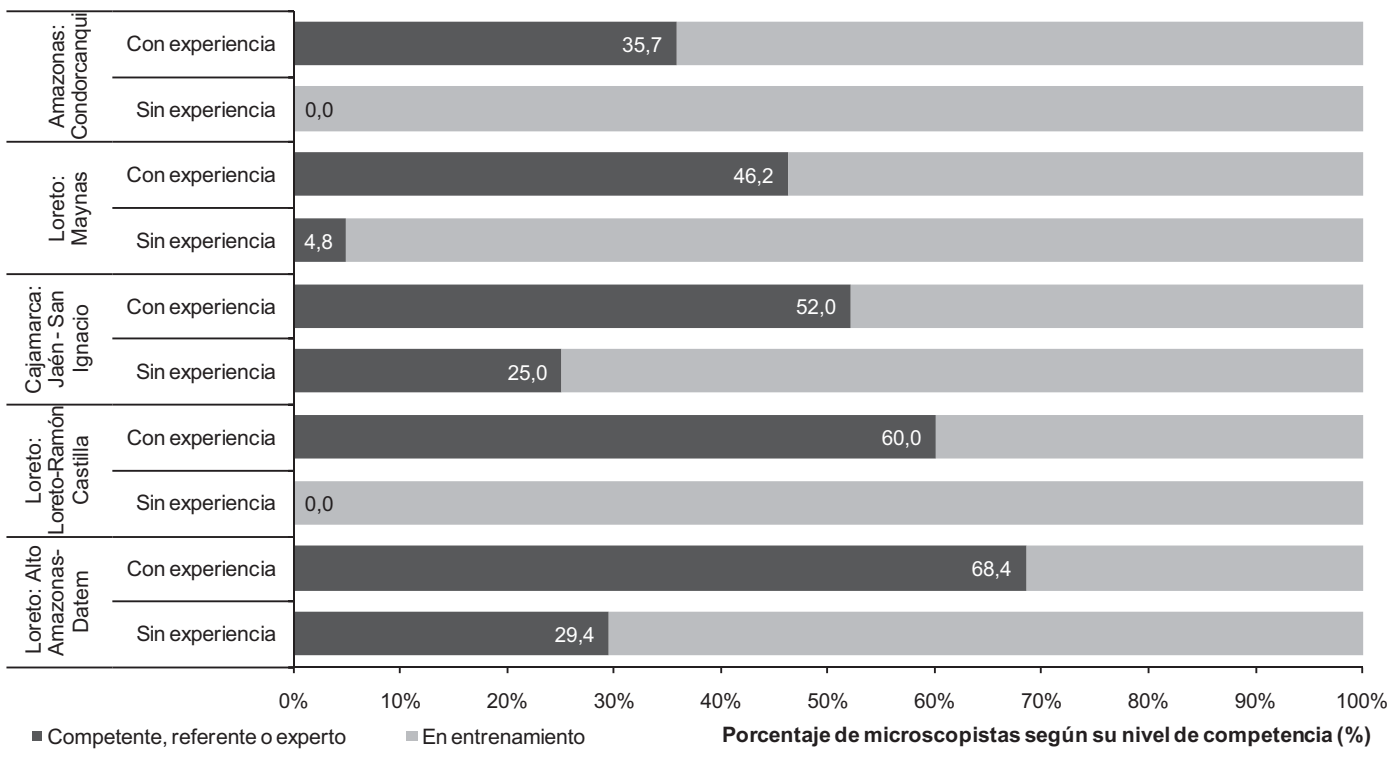

Figura 2. Clasificación de los microscopistas según su competencia por provincias en la Amazonía peruana, 2007. 
Tabla 3. Clasificación de los microscopistas según su competencia en la Amazonía Peruana, 2007.

\begin{tabular}{|c|c|c|c|c|c|c|}
\hline \multirow{2}{*}{ Clasificación según nivel de concordancia } & \multicolumn{2}{|c|}{$\begin{array}{l}\text { Microscopista sin } \\
\text { experiencia }\end{array}$} & \multicolumn{2}{|c|}{$\begin{array}{c}\text { Microscopista con } \\
\text { experiencia }\end{array}$} & \multicolumn{2}{|c|}{ Total microscopistas } \\
\hline & $\mathrm{N}=68$ & (\%) & $\mathrm{N}=76$ & (\%) & $\mathrm{N}=144$ & (\%) \\
\hline En entrenamiento $<70 \%$ & 60 & $(88,2)$ & 36 & $(47,4)$ & 96 & $(66,7)$ \\
\hline Competente 70 a $<80 \%$ & 5 & $(7,4)$ & 14 & $(18,4)$ & 19 & $(13,2)$ \\
\hline Referente 80 a $<90 \%$ & 2 & $(2,9)$ & 17 & $(22,4)$ & 19 & $(13,2)$ \\
\hline Experto $\geq 90 \%$ & 1 & $(1,5)$ & 9 & $(11,8)$ & 10 & $(6,9)$ \\
\hline
\end{tabular}

qué tipo de láminas tienen un menor porcentaje de acierto (Tabla 4). La concordancia en microscopistas con experiencia fue: $85,1 \%$ para láminas negativas; $77,1 \%$ para el total de láminas $P$. vivax; $70,1 \%$ para láminas $P$. vivax con densidad parasitaria menor de 500 parásitos/ $\mu \mathrm{L} ; 67,7 \%$ para el total de láminas $P$. falciparum; $49,2 \%$ para $P$. falciparum $<500$ parásitos $/ \mu \mathrm{L} ; 46,9 \%$ para $P$. malariae y $25,0 \%$ para malaria mixta ( $P$. falciparum y $P$. vivax). La concordancia en microscopistas sin experiencia fue: $61,5 \%$ para láminas negativas; $61,7 \%$ para $P$. vivax; $44,3 \%$ para $P$. vivax $<500$ parásitos $/ \mu \mathrm{L}$; $44,2 \%$ para $P$. falciparum; $24,7 \%$ para $P$. falciparum $<500$ parásitos $/ \mu \mathrm{L} ; 24,6 \%$ para $P$. malariae y $9,6 \%$ para malaria mixta ( $P$. falciparum y $P$. vivax).

En total, solo el $17,7 \%$ de los microscopistas diagnosticaron correctamente las láminas mixtas, la mayoría de ellos las diagnosticaron como: P. vivax (43,1\%), negativo (18,3\%) y $P$. falciparum (16,7\%). Los porcentajes fueron similares en microscopistas sin y con experiencia.

\section{DISCUSIÓN}

En el presente artículo se comparten los resultados de la primera evaluación de competencias de los microscopistas de establecimientos de primer nivel de atención en el Perú usando paneles de láminas estandarizadas, siguiendo las recomendaciones de la OMS ${ }^{(11)}$. Laevaluación oacreditación de las competencias del personal a cargo del diagnóstico de la malaria es considerado por la OMS como un pilar importante dentro del sistema de aseguramiento de calidad del diagnóstico de la malaria ${ }^{(12)}$. Si bien el sistema nacional de control de calidad del diagnóstico microscópico ha incorporado una metodología similar basada en el envío de paneles de láminas estandarizadas para el control de calidad externo de laboratorios de referencia regional como complemento al control de calidad indirecto usual (15-17), la evaluación de competencias en el diagnóstico microscópico en el primer nivel de atención usando dichos paneles no ha sido considerada.

Se evidencia dificultades en los microscopistas para la realización de un correcto diagnóstico de la malaria; siendo estas mayores en microscopistas recientemente capacitados y con poca experiencia, y particularmente en la identificación de malaria mixta, malaria por $P$. malariae y malaria con densidades parasitarias bajas.

Diversos estudios han demostrado cómo la selección de la persona a entrenar, el tipo y periodicidad del entrena-

Tabla 4. Concordancia según tipo de lámina en la Amazonía peruana, 2007.

\begin{tabular}{|c|c|c|c|c|c|c|c|c|c|}
\hline \multirow{3}{*}{$\begin{array}{l}\text { Diagnóstico } \\
\text { Características } \\
\text { de las láminas }\end{array}$} & \multicolumn{3}{|c|}{ Microscopista sin experiencia } & \multicolumn{3}{|c|}{ Microscopista con experiencia } & \multicolumn{3}{|c|}{ Total microscopistas } \\
\hline & \multirow{2}{*}{$\mathbf{N}$} & \multicolumn{2}{|c|}{ Concordancia (\%) } & \multirow{2}{*}{$\mathbf{N}$} & \multicolumn{2}{|c|}{ Concordancia (\%) } & \multirow{2}{*}{$\mathbf{N}$} & \multicolumn{2}{|c|}{ Concordancia (\%) } \\
\hline & & Media & Mediana & & Media & Mediana & & Media & Mediana \\
\hline \multicolumn{10}{|l|}{ Negativos } \\
\hline Todas & 68 & 61,5 & 60,0 & 76 & 85,1 & 100,0 & 144 & 73,9 & 80,0 \\
\hline \multicolumn{10}{|l|}{ P. vivax } \\
\hline Todas & 68 & 61,7 & 60,0 & 76 & 77,1 & 80,0 & 144 & 69,8 & 66,7 \\
\hline$<500 \mathrm{par} / \mu \mathrm{L}$ & 56 & 44,3 & 41,7 & 63 & 70,1 & 100,0 & 119 & 58,0 & 50,0 \\
\hline \multicolumn{10}{|l|}{ P. falciparum } \\
\hline Todas & 68 & 44,2 & 40,0 & 76 & 67,7 & 66,7 & 144 & 56,6 & 60,0 \\
\hline$<500$ par/ $\mu \mathrm{L}$ & 54 & 24,7 & 0,0 & 62 & 49,2 & 50,0 & 116 & 37,8 & 33,3 \\
\hline \multicolumn{10}{|l|}{ P. malariae } \\
\hline$>=500 \mathrm{par} / \mu \mathrm{L}$ & 59 & 24,6 & 0,0 & 65 & 46,9 & 50,0 & 124 & 36,3 & 0,0 \\
\hline \multicolumn{10}{|c|}{ Malaria mixta (P. falciparum y $P$. vivax) } \\
\hline Todas & 68 & 9,6 & 0,0 & 76 & 25,0 & 0,0 & 144 & 17,7 & 0,0 \\
\hline
\end{tabular}


miento y la experiencia y práctica, van a ser decisivos en el diagnóstico apropiado de un paciente con malaria ${ }^{(12,18)}$. La OMS da pautas de cómo y por cuánto tiempo debe ser el entrenamiento ${ }^{(13)}$, diferenciando para ello dos grupos: aquellos que no tienen experiencia previa y aquellos que vienen realizando la labor de microscopistas. En los primeros se sugiere la realización de una evaluación de la vista, requisito que difícilmente puede cumplirse en algunos lugares periféricos de la Amazonía peruana.

La experiencia tiene relación con el tiempo en años que tiene el microscopista realizando la lectura microscópica, pero también con la cantidad y variedad (tipos de parásitos) de láminas positivas que lee periódicamente (mayor práctica en la lectura). Esta afirmación podría explicar por qué las medias de concordancia y proporción con competencia aceptable (competente, referente o experto) son más altas en los microscopistas con experiencia, justo de las provincias del departamento de Loreto, caracterizadas por contar con la mayor concentración de casos en el país (Maynas, Alto Amazonas -Datem, Loreto-Ramón Castilla).

Resulta obvio de que el resultado de la evaluación de competencias, además de la experiencia del microscopista, depende de la mayor o menor dificultad de las láminas. El análisis por cada lámina del panel en los microscopistas con experiencia demostró una concordancia aceptable para las láminas con $P$. vivax ( $P$. vivax todas: $77,1 \%, P$. vivax 500 parásitos $/ \mu \mathrm{L}: 70,1 \%$ ) y también aceptable para las láminas de $P$. falciparum $(67,7 \%)$, con algunas diferencias según provincia. Es necesario recordar que los lugares donde brindan sus servicios los microscopistas evaluados son considerados áreas de alto riesgo de transmisión de malaria en donde predomina la malaria por $P$. vivax (P.vivax: $79,8 \%$, P. falciparum $20,1 \%$ ).

En cambio, la concordancia de los microscopistas con experiencia fue pobre para las láminas de $P$. malariae $(46,9 \%)$, láminas mixtas $(25,0 \%)$ y láminas $P$. falciparum con densidad parasitaria menor de 500 parásitos/ $\mu \mathrm{L}$ $(49,2 \%)$. Al respecto, Maguire et al. demostraron que aun en microscopistas considerados "expertos" los niveles de concordancia con láminas estándares (corroboradas por PCR) estuvieron asociados con la dificultad de la lámina bajo evaluación; siendo las láminas con baja parasitemia ${ }^{(19)}$, aquellas con parásitos encontrados con menos frecuencia ( $P$. malariae) y aquellas con infecciones mixtas consideradas como las más difíciles (14). En dicho estudio aproximadamente 15\% falló para detectar parásitos cuando las densidades parasitarias fueron menores de 100 parásitos/ $\mu \mathrm{L}$ y solo $47 \%$ (rango $13-67 \%$ ) logró diagnosticar infecciones mixtas. En forma similar, Milne et al. encontraron que las infecciones únicas podrían ser mal diagnosticadas hasta en un
$25 \%$ de las veces y que los diagnósticos incorrectos en infecciones mixtas eran aun más frecuentes, $71 \%{ }^{(18)}$.

Las estadísticas peruanas reportan malaria mixta ( $P$. vivax y $P$. falciparum) en porcentajes mínimos $(<0,1 \%)$; sin embargo, es posible que la incidencia de casos mixtos sea mucho mayor, y que el error de los microscopistas en la identificación sea la causa del pobre reporte. Nuestro estudio encontró que la mayoría de casos de malaria mixta fueron diagnosticados como $P$. vivax y negativos, lo cual es preocupante ya que se estaría dejando sin tratamiento apropiado y oportuno a pacientes con presencia del parásito $P$. falciparum en su sangre, con el correspondiente riesgo de complicaciones y muerte por ello.

Para la interpretación correcta de nuestros resultados es importante tener en cuenta algunas consideraciones. Primero, en el presente estudio no se evaluó la competencia global de los microscopistas, sino aquella relacionada con su capacidad de lectura de láminas. La competencia global incluye además, la capacidad y habilidad para tomar la muestra de sangre, extender, fijar y preparar la lámina previa a su lectura. Segundo, fue necesario adaptar los paneles de láminas recomendados por la OMS con el reemplazo de las láminas de $P$. ovale y las láminas de presentación inusual por láminas con $P$. vivax, $P$. falciparum o malaria mixta, ya que en el país nunca ha sido reportado caso alguno de malaria por dicha especie de Plasmodium. Tercero, si bien un examen cuidadoso de la vista sin alteraciones es un requisito imprescindible al momento de seleccionar o ratificar a las personas que estarán a cargo del diagnóstico microscópico a nivel nacional, no podemos asegurar que los microscopistas evaluados hayan cumplido dichos requisitos.

Si bien los niveles de concordancia y proporción de microscopistas con competencia aceptable (competentes, referentes o expertos) no son óptimos usando los estándares propuestos por OMS ${ }^{(4,6)}$ inclusive en microscopistas con experiencia, esta primera evaluación nos ayuda a trazar una línea de base sobre la cual trabajar, teniendo como marco un sistema de aseguramiento de la calidad. La información obtenida de la evaluación sirvió para tomar decisiones inmediatas como la evaluación de la vista, el reentrenamiento de casi la totalidad de los microscopistas sin experiencia y su seguimiento cercano a través del control de calidad del diagnóstico microscópico rutinario que realizan en sus establecimientos de salud (monitoreo del desempeño periódico). En los microscopistas con experiencia que tuvieron niveles de concordancia no aceptables es necesario realizar una nueva evaluación de competencias para definir su continuidad, previo examen de la vista, un 
reentrenamiento de corta duración y el seguimiento del desempeño por un periodo no menor de seis meses.

En el marco de un mejoramiento continuo de la calidad, debe tenerse en cuenta que el desempeño final de los microscopistas en los establecimientos de salud en la Amazonía peruana, no solo está influenciado por su competencia, sino también por otros factores como las condiciones de trabajo, su carga laboral y los equipos e insumos apropiados ${ }^{(13)}$. La OMS recomienda que la evaluación o acreditación periódica de la competencia debe formar parte de este proceso de mejora del desempeño, siendo necesario para ello: una normativa para su inclusión en el sistema de gestión de calidad del diagnóstico microscópico nacional, un programa de entrenamiento continuo para mejorar la competencia y alianzas con universidades $u$ otras instituciones que sean fuente productora de láminas estandarizadas que puedan ser usadas en el largo plazo ${ }^{(20-22)}$.

\section{Financiamiento}

La investigación contó con el apoyo del Organismo Andino de Salud- Convenio Hipólito Unanue (ORASCONHU) en el marco del Proyecto "Control de la Malaria en las Zonas Fronterizas de la Región Andina: Un Enfoque Comunitario" - PAMAFRO, cuyo financiamiento es del Fondo Mundial de Lucha contra el VIH/SIDA, Tuberculosis y Malaria (Grant MAA-305-G01-M).

\section{Conflictos de Interés}

Los autores declaran no tener conflictos de interés en la ejecución y publicación de este artículo.

\section{REFERENCIAS BIBLIOGRÁFICAS}

1. Organización Mundial de la Salud. Informe sobre la salud en el mundo, 2007: un porvenir más seguro. Protección de la salud pública mundial en el siglo XXI. Ginebra: OMS; 2007.

2. Perú, Ministerio de Salud. Análisis de la situación de salud del Perú 2005. Lima: MINSA; 2006.

3. Chapilliquen F. Tendencia y situación de las enfermedades sujetas a vigilancia epidemiológica: malaria. Bol Epidemiol (Lima). 2007;16(52):716-17.

4. World Health Organization. Malaria epidemics: forecasting, prevention, early detection and control. From policy to practice. Geneva: WHO; 2004.

5. World Health Organization. Strategic orientation paper on prevention and control of malaria. Geneva: WHO; 2005.

6. Gatti S, Gramegna M, Bissofi Z, Raglio A, Gulleta M, Klersy C, et al. A comparison of three diagnostic techniques for malaria: a rapid diagnostic test (NOWH Malaria), PCR and microscopy. Ann Trop Med Parasitol. 2007;101(3):195-204.

7. Chilton D, Malik AN, Armstrong M, Kettelhut M, ParkerWilliams J, Chiodini PL. Use of rapid diagnostic test for diagnosis of malaria in the UK. J Clin Pathol 2006;59(8):862-66.
8. Wongsrichanalai C, Barkus MJ, Muth S, Sutamihardja A, Wernsdorfer W. A review of malaria diagnostic tools: microscopy and rapid diagnostic test (RDT). Am J Trop Med Hyg. 2007;77(6 Suppl):119-27.

9. Instituto Nacional de Salud. Manual de procedimientos del laboratorio para el diagnóstico de malaria. Lima: INS; 2003.

10. Payne D. Use and limitations of light microscopy for diagnosing malaria at the primary health care level. Bull World Health Organ. 1988;66(5):621-26.

11. World Health Organization. Informal consultation on quality control of malaria microscopy. Geneva: WHO; 2006.

12. World Health Organization. Malaria light microscopy creating a culture of quality. Report of WHO SEARO/WPRO workshop on quality assurance for malaria microscopy. Geneva: WHO; 2005.

13. Organización Panamericana de la Salud. Guía para la implementación de un sistema de gestión de calidad en el diagnóstico microscópico de malaria: Estandarización de procedimientos y herramientas sobre el control de calidad y la evaluación externa del desempeño en las redes de laboratorio. Caracas: OPS; 2004.

14. Maguire JD, Ledermann ER, Barcus MJ, O'Meara WA, Jordon RG, Duong S, et al. Production and validation of durable, high quality standardized malaria microscopy slides for teaching, testing and quality assurance during an era of declining diagnostic proficiency. Malaria J. 2006;5:92.

15. Perú, Ministerio de Salud. NTS N. ${ }^{\circ} 082-M I N S A / I N S-$ v.01. Norma técnica de salud para el control de calidad del diagnóstico microscópico de malaria. Lima: MINSA; 2010.

16. Gutierrez S, Arrospide N. Assessment of the external quality control of parasitological diagnostic malaria in Peru. Am J Trop Med Hyg. 2004;71(Suppl 4):211.

17. Gutierrez S, Arrospide N. Evaluación externa de la calidad del diagnóstico microscópico de malaria mediante láminas panel. En: Instituto Nacional de Salud. IV Congreso Científico Internacional del Instituto Nacional de Salud: Libro de Resúmenes. Lima: INS; 2010. p.52.

18. Milne LM, Kyi MS, Chiodini PL, Warhurst DC. Accuracy of routine laboratory diagnosis of malaria in the United Kingdom. J Clin Pathol. 1994;47(8):740-42.

19. Mc Kenzie E, Sirichaisinthop J, Miller S, Gasser RA, Wongsrichanalai C. Dependence of malaria detection and species diagnosis by microscopy on parasite density. Am J Trop Med Hyg. 2003;69(4):372-76.

20. Bell D, Wongsrichanalai C, Barnwell JW. Ensuring quality and access for malaria diagnosis: how can it be achieved? Nat Rev Microbiol. 2006;4(9):682-95.

21. Church DL, Lichtenfeld A, Elsayed S, Kuhn S, Gregson D. A regional centralized microbiology service in calgary for the rapid diagnosis of malaria. Arch Pathol Lab Med. 2003;127(6): 687-93.

22. Ohrt C, Obare P, Nanakorn A, Adhiambo C, Awuondo K, O'Meara WP, et al. Establishing a malaria diagnostics centre of excellence in Kisumu, Kenya. Malaria J. 2007;6:79.

Correspondencia: Angel Rosas-Aguirre

Dirección: Jr. Aldebarán 320, G-301. Urb. Polo Hunt. Surco, Lima 33 - Perú.

Teléfono: (511) 436-8690 / (51) 997-347-904

Correo electrónico: angelrosasa@gmail.com 